\title{
Clinical Evaluation of Stretchable and Wearable Inkjet-Printed Strain Gauge Sensor For Respiratory Rate Monitoring At Different Measurements Locations
}

\author{
Ala'aldeen Al-Halhouli ${ }^{1,2,3,}{ }^{*}$, Loiy Al-Ghussain ${ }^{1,4}$, Saleem El Bouri ${ }^{1}$, Haipeng Liu ${ }^{5}$, Dingchang \\ Zheng $^{5}$ \\ ${ }^{1}$ Mechatronics Engineering Department/ NanoLab, School of Applied Technical Sciences, \\ German Jordanian University, P.O. Box 35247, Amman 11180, Jordan \\ ${ }^{2}$ Institute of Microtechnology, Technische Universität Braunschweig, Braunschweig, Germany \\ ${ }^{3}$ Faculty of Engineering, Middle East University, Amman 11831, Jordan \\ ${ }^{4}$ Mechanical Engineering Department, University of Kentucky, Lexington, KY 40506, USA \\ ${ }^{5}$ Medical Device and Technology Research Laboratory, School of Allied Health, Faculty of \\ Health, Education, Medicine and Social Care, Anglia Ruskin University, Chelmsford CM1 1SQ, \\ UK \\ *Correspondence: alaaldeen.alhalhouli@gju.edu.jo
}

\begin{abstract}
Introduction: The respiration rate (RR) is a vital sign in physiological measurement and clinical diagnosis. RR can be measured using stretchable and wearable strain gauge sensors which detect the respiratory movements in the abdomen or thorax areas caused by volumetric changes. In different body locations, the accuracy of RR detection might differ due to different respiratory movement amplitudes. Few studies have quantitatively investigated the effect of the measurement location on the accuracy of new sensors in RR detection.

Methods: Using a stretchable and wearable inkjet-printed strain gauge (IPSG) sensor, RR was measured from five body locations (umbilicus, upper abdomen, xiphoid process, upper thorax, and diagonal) on thirty healthy test subjects while sitting on an armless chair. At each location, reference RR was simultaneously detected by the e-Health sensor, and the measurement was repeated twice. Subjects were asked about the comfortableness of locations. Based on Levene's test, ANOVA was performed to investigate if there is a significant difference in RR between sensors, measurement locations, and two repeated measurements. Bland-Altman analysis was
\end{abstract}


applied to the RR measurements at different locations. The effects of measurement site and measurement trials on RR difference between sensors were also investigated.

Results: There was no significant difference between IPSG and reference sensors, between any locations, and between the two measurements (all $\mathrm{p}>0.05$ ). As to the RR deviation between IPSG and reference sensors, there was no significant difference between any locations, or between two measurements (all p>0.05). All the thirty subjects agreed that diagonal and upper thorax positions were the most uncomfortable and most comfortable locations for measurement, respectively.

Conclusion: The IPSG sensor could accurately detect RR at five different locations with good repeatability. Upper thorax was the most comfortable location.

Keywords: Wearable Sensors, Clinical Evaluation, Respiratory Rate, Inkjet Printing, PDMS

\section{Introduction}

Respiratory rate (RR) is commonly defined as the times of respiration in a minute, with the unit of breath per minute (BPM). In physiological measurement and clinical monitoring, RR is a vital sign where the RR variations could reflect the state of cardiorespiratory system and related diseases [1] [2], such as cardiac arrest [2], [3] and respiratory failure [2]. Despite its clinical importance, RR is often neglected or measured inaccurately by the manual counting of nursing staff [4], [5]. The traditional RR monitoring devices such as spirometers and capnography [6] are uncomfortable to wear and limit patients' mobility.. There is an urgent need for more wearable and comfortable RR sensors.

Recently, some stretchable and wearable sensors have been developed to measure RR continuously and automatically [7]-[10] based on the detection of volumetric changes in the ribcage or abdomen areas [11], [12]. Stretchable and wearable sensors are convenient to use during daily activities [13]-[17]. Additionally, flexible and stretchable sensors are comfortable sensors to achieve continuous physiological monitoring. The stretchability and flexibility give these sensors skin-like characteristics therefore the patients will more likely tolerate these sensors [13]. Therefore, these sensors provide the possibility of continuous, convenient, and comfortable RR monitoring. 
With stretchable and wearable sensors, RR could be measured in different body locations. Liu et al. [18] assessed the validity of a newly developed wearable strain sensor for respiratory movements at four different locations namely axilla, xiphoid process, $10^{\text {th }}$ rib and umbilicus on 21 subjects. At each location they mounted three wearable sensors at the left, right and middle to measure the amount of change in the circumference during inhalation and exhalation, with a reference tape sensor recording the respiratory movement. Regarding the results repeatedly measured by wearable sensors, the Intraclass Correlation Coefficient (ICC) values for intra-rater reliability is high (range of ICC: 0.94 to 0.98 ) in all the four locations. The largest circumference change was observed from the wearable sensor in the middle of the $10^{\text {th }}$ rid (mean $\pm S D$ : $0.6 \pm 0.19 \mathrm{~cm}$ ) where the correlation between respiratory movements measured by the wearable sensor and reference tape sensor was the largest $(0.85, \mathrm{p}<0.05)$. Furthermore, Furtak et al. [19] developed a strain sensor for RR monitoring using screen printing technique. Carbon nanotube paste was deposited on a textile substrate, then attached to two different shirts using two different stitching configurations. They used two sensors placed on the chest (bust) and at the under-bust region for each test. The results indicate that there is no significant difference in RR measurements between the two locations with 0.6 BPM deviation using the best sensor configuration. Furthermore, in Leicht et al. [20] proposed the integration of magnetic induction sensor in the diagonal location in safety belts for RR monitoring during driving.

Due to the different amplitudes in respiratory movement, different locations might differ in the accuracy of RR monitoring. It has been reported that the accuracy of RR measurements using strain gauge sensors changes with different body locations [18], [19]. However, the majority of existing studies on RR monitoring accuracy were focused at two common locations namely thorax [21]-[24] and abdomen [21], [23], [25]. Diagonal mounting of RR sensors [9], [20] was also investigated. However, there is a lack of studies investigating the accuracy of stretchable and wearable RR strain sensors at more than three positions on human body. Moreover, most of the existing studies on the accuracy of RR sensors at different locations did not include the validation of sensor repeatability [12], [19], [21]-[25].

To investigate if RR measurement using the stretchable sensor is repeatable at different measurement locations, and to investigate the difference in measurement accuracy between 
locations, this study aims to preliminarily validate the performance and the functionality of a stretchable and wearable inkjet-printed strain gauge (IPSG) at five different body locations.

\section{Methodology}

\subsection{Physiological Measurements}

\subsubsection{Subjects}

Thirty test subjects (six females and 24 males) with an age deviation of $26.67 \pm 6.23$ years (mean \pm SD) under normal breathing rhythm without any known respiratory diseases participated in this study with written informed consent. The subjects were given a brief of the procedure at the beginning of the experiment and reminders during the experiment [26].

\subsubsection{Sensors}

The IPSG RR sensor was fabricated using inkjet printing technology on Polydimethylsiloxane (PDMS) substrate [27]. Inkjet printing is an emerging fabrication technique that has been used extensively in the development of wearable sensors [28]-[30]. The sensor employs the volume change of either the ribcage or the abdomen in RR detection where the inductance of the sensor increases during the inhalation process while it decreases while the exhalation. The strain gauge sensor was implemented in Wheatstone bridge circuit. The output signal from the developed sensor was processed in a microcontroller and transferred to a computer where the sensor has the ability to provide real-time monitoring.

The results obtained from the developed sensor are compared with the results from the reference nasal e-Health sensor (e-Health AirFlow sensor, Cooking Hacks) which was validated by several studies in the literature [31]-[34]. The e-Health sensor detects the variation in temperature of the nasal airflow. Liu et al. [35] mentioned that airflow measurement is deemed as a more reliable method compared with the methods using respiratory movement or respiratory modulation. Figure 1 shows a sample of the RR signals measured by the IPSG and e-Health sensors, note that the phase shift in the RR signals is due to the working principle of each sensor. The peaks in the e-Health sensor signal were formulated during the exhale during which the airflow temperature is high while the peaks in the IPSG sensor signal are formulated during the inhale. 


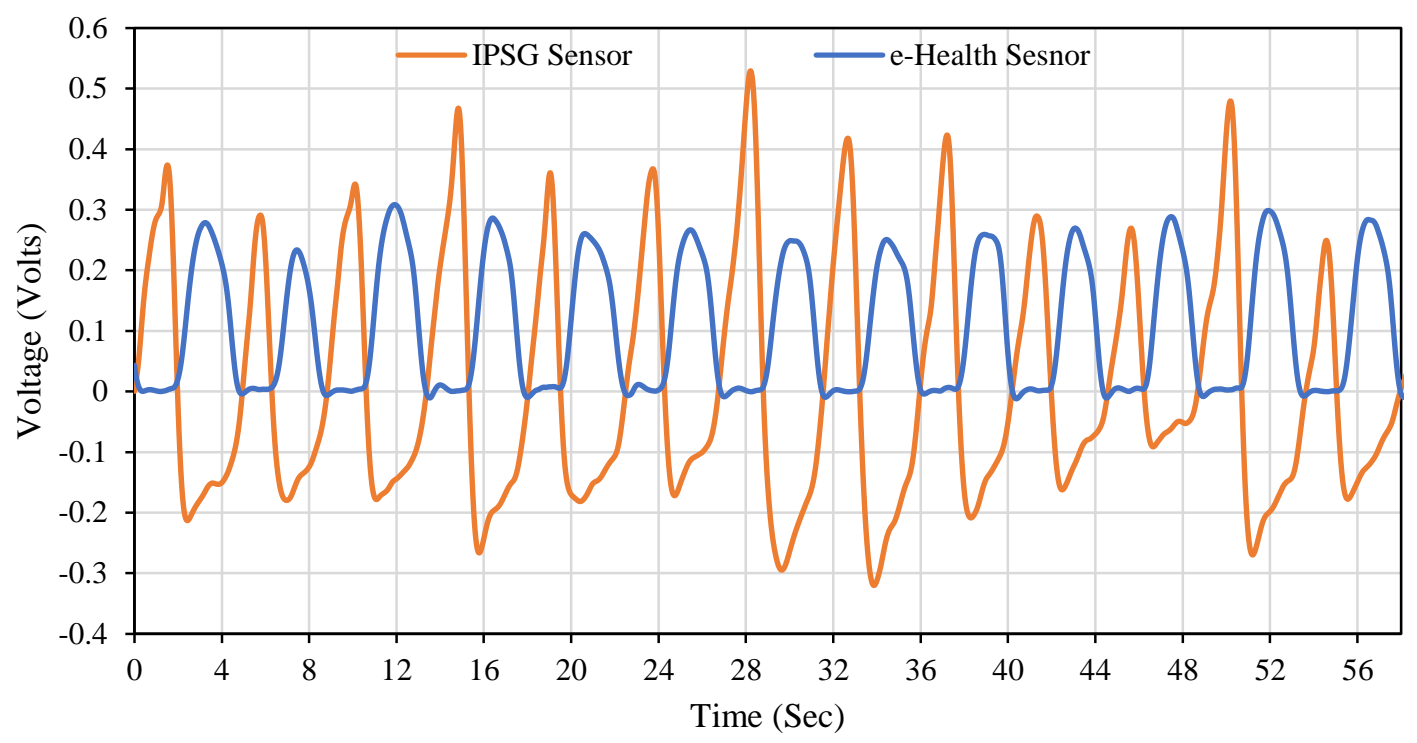

Figure 1. Sample of the RR signal from the IPSG and e-Health sensors.

\subsubsection{Measurement procedure}

In this study, the IPSG sensor was tested at five different locations: umbilicus (navel), upper abdomen, xiphoid process, upper part of thorax and diagonal from the shoulder to the end of the ribcage as shown in Figure 2. The subjects were asked to sit on armless wooden chair facing the wall in an office room [36]. In each location, the test was repeated twice in order to investigate the repeatability where in each trial the RR readings were recorded for one minute with a $30 \mathrm{~s}$ break between the two trials. Note that the order of tested locations was selected randomly. Figure 3 shows the measuring protocol used to inspect the effect of the measurement location on the accuracy of the sensor. Finally, after each test the test subjects were asked about the most comfortable location of measurement.

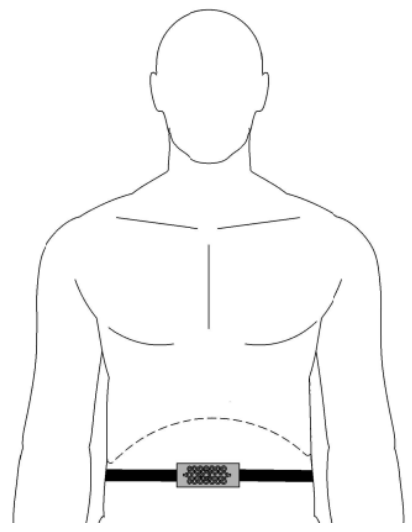

(a)

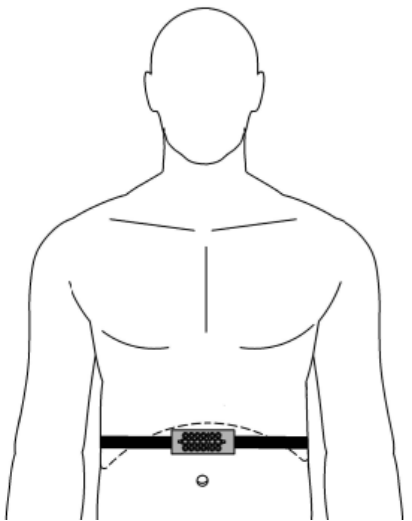

(b)

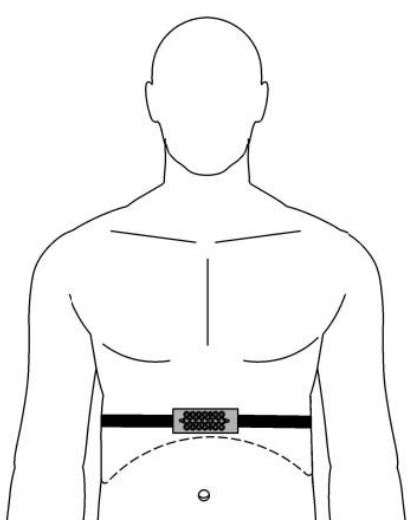

(c) 


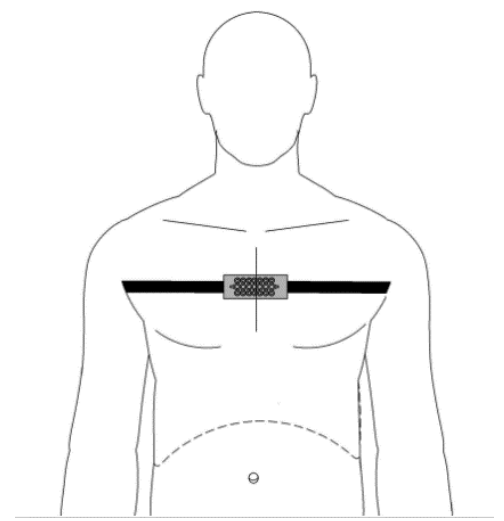

(d)

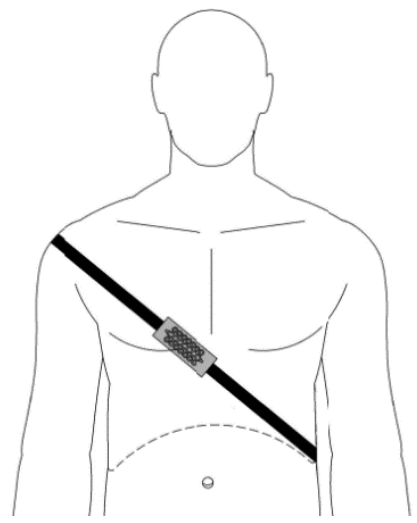

(e)

Figure 2. The sensor's position on human body: a) umbilicus, b) upper abdomen, c) thorax at xiphoid process, d) upper thorax and e) diagonal (from left shoulder to the thorax right end). The dotted line represents the end of the ribcage.
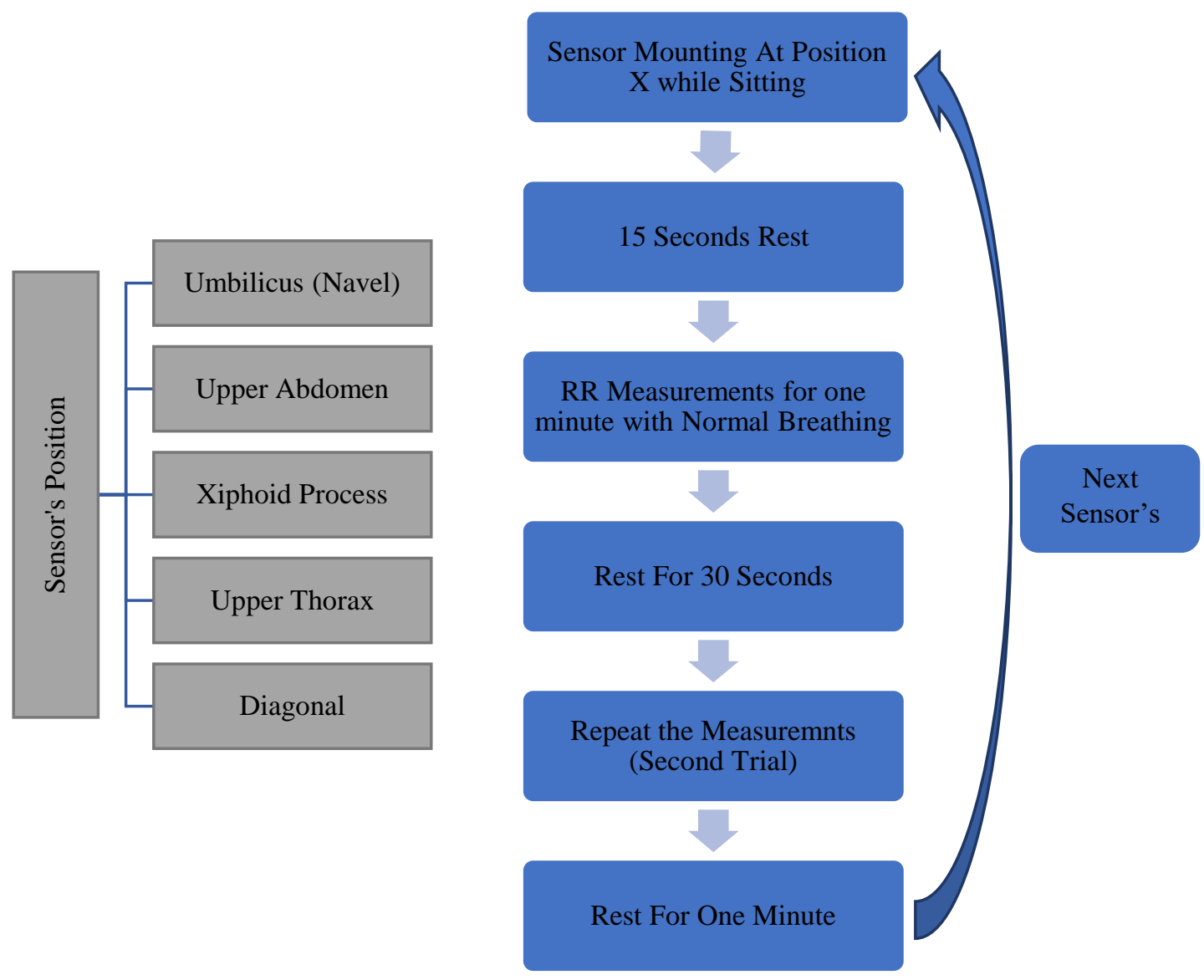

Figure 3. The adopted measurements protocol [26]. 


\subsection{Extraction of RR}

The signal processing was performed using MATLAB Simulink (R2018b, MathWorks, Natick, MA, USA). The algorithm starts with acquiring raw data from the sensor at a sampling frequency of $100 \mathrm{~Hz}$ as shown in Figure 4 where the respiration frequency is the peak in frequency spectrum. It should be noted that the FFT implementation was set to the auto implementation mode with 10s window size.

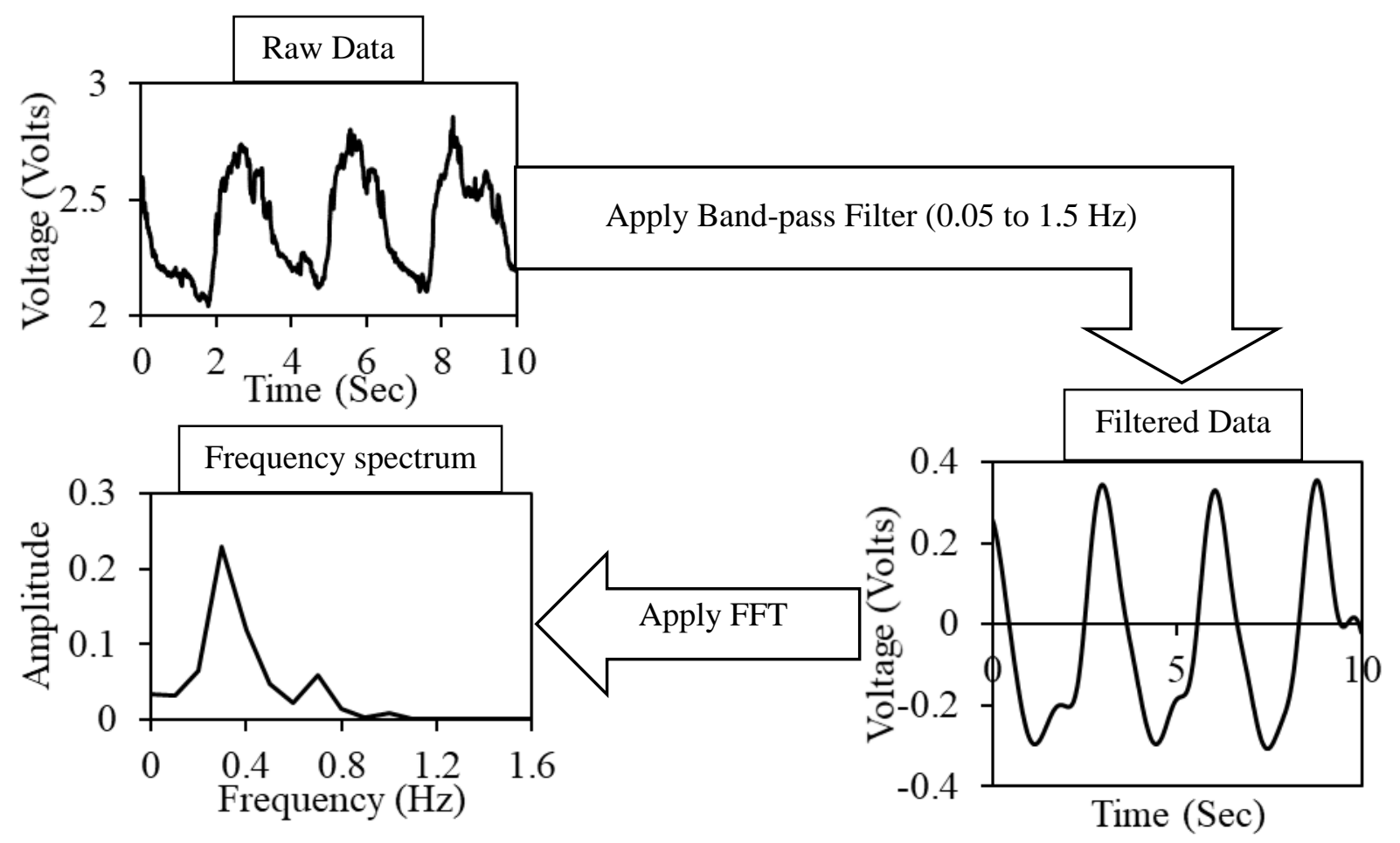

Figure 4. Derivation flow chart of respiration rate from the RR sensor.

\subsection{Statistical Analysis}

\subsubsection{RR Measurement: Effects of Measurements Trials, Sites, and Sensors}

Analysis Of Variance (ANOVA) test was performed on SPSS (SPSS 20.0, SPSS Inc., Chicago, Illinois, USA) to evaluate the existence of significant statistical difference between the measurements trials and the statistical difference between the sensor's readings at different locations as well as the readings of the IPSG and reference sensor. In order to properly use ANOVA, the normality and the homogeneity of variance (equality of variance) should be inspected however since the sample had more than 25 observations (central limit theorem) there was no need to inspect the normality of it [37]. It should be noted that the homogeneity of variance was inspected using Levene's test on Minitab (Minitab 16, Minitab Inc., State College, 
Pennsylvania, USA) which is one of the common tests used in the evaluation of the homogeneity of variance.

\subsubsection{RR Deviation: Effects of Measurements Trials and Sites}

For each measurement, RR deviation was calculated as the RR measured by the IPSG sensor minus the reference RR. To investigate if the RR deviation is repeatable and independent from measurement location, ANOVA with Levene's test was applied to the RR deviation data.

\subsubsection{Bland-Altman analysis}

Bland-Altman analysis was used to evaluate the accuracy of the IPSG sensor at the five locations where Bland-Altman analysis was applied on RR measurements dataset from the two trails. Bland-Altman analysis compares the results obtained from two measuring devices or techniques where it represents the agreement between the two measuring techniques.

\subsubsection{Linear Regression}

To evaluate the correlation between the measured RRs by the IPSG and e-Health sensors and to specify if the RR reading follows a linear correlation, the RR measurements dataset from the two trails were fitted to a linear regression model. The correlation was evaluated based on the regression coefficient which reflects the consistency of the change in the RR measured by the IPSG sensor and the change in RR measured by the reference sensor. Moreover, p-values were used to judge the statistical significance of the correlation components where coefficients with $\mathrm{p}$ values larger than the significance level (0.05) have no statistical significance on the RR correlation (null hypothesis is rejected).

\section{Results}

\subsection{Repeatability of RR Measurements}

Levene's test indicates that the dataset satisfies the homogeneity of variance $(\mathrm{p}=0.86)$. Knowing that the dataset satisfies the homogeneity of variance criteria, ANOVA test can be applied on the dataset to inspect the statistical significance between the measurements' trials, measurements locations and measurements source. The ANOVA test applied on the RR dataset indicates that there is no significant statistical variance between the measurement's trials, measurements locations and measurements source where the p-value in all the cases in was larger than the significance level (0.05). 
The respiratory rate had high individual difference due to several factors such as gender, health status and age. Moreover, the amount of volume change during respiration depends on the measurement location on human body. The measured respiration rate of the thirty test subjects by the IPSG sensor varies between 7.8 BPM and 39.06 BPM. The RRs measured by the IPSG and reference sensors are not significantly different at the five different locations, and for the two measurements trials.

\subsection{The Effect of The Measurement Location on RR}

The functionality and the accuracy of the IPSG sensors depend on the amount of force applied to it. The force depends on the amount of volume change which causes different respiratory movements in different locations [18]. The stretchability and wearability of the IPSG sensors make them more vulnerable to the amount of volume change during the respiration process at different locations in the abdomen and thorax areas. ANOVA test applied to the deviation results concluded that there was no statistical significance between the measurements locations and trials. Figure 5 shows the average deviation in the respiration rate measured by IPSG sensor compared with the e-Health sensor at the five measurement's locations, while Figure 6 shows the Bland-Altman analysis for the measured respiratory rate at five measurement's locations.

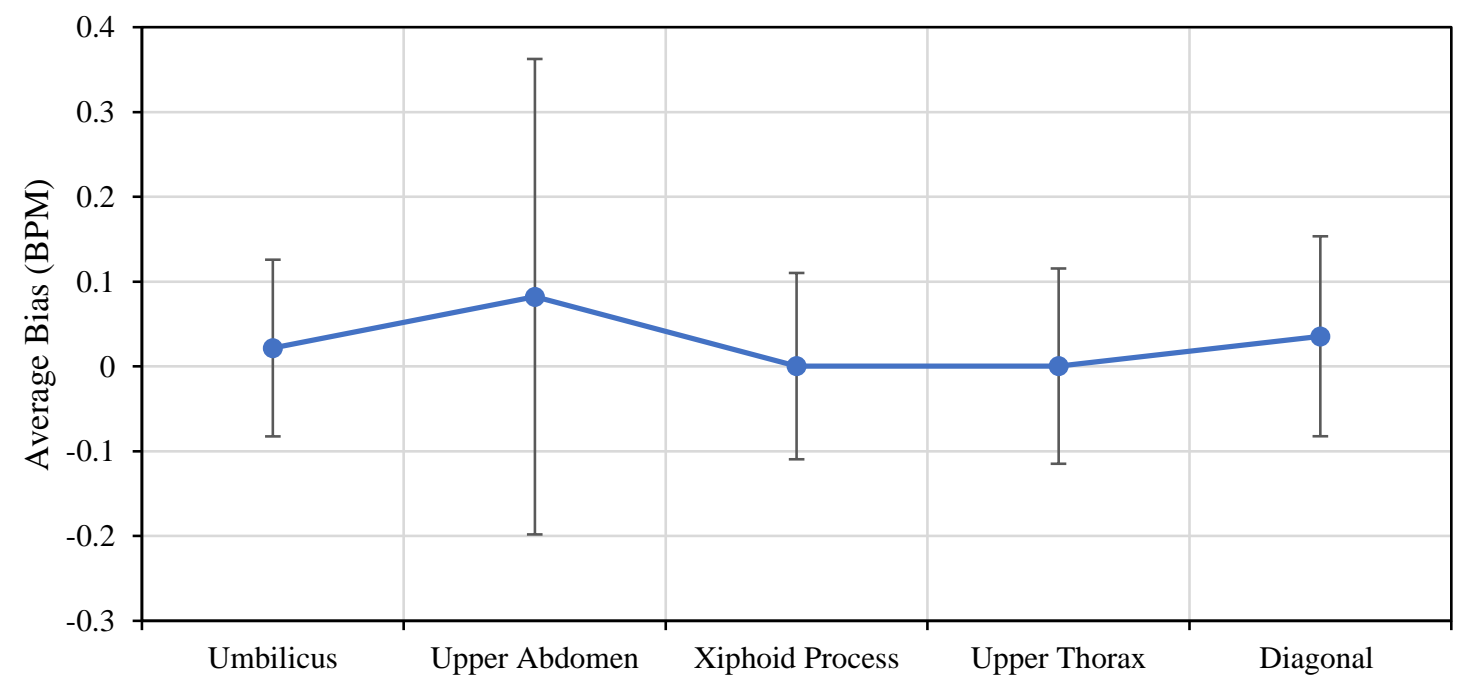

Figure 5. Average respiration rate deviation of the two trials compared with the reference sensor for the thirty test subjects at different measurements locations. 

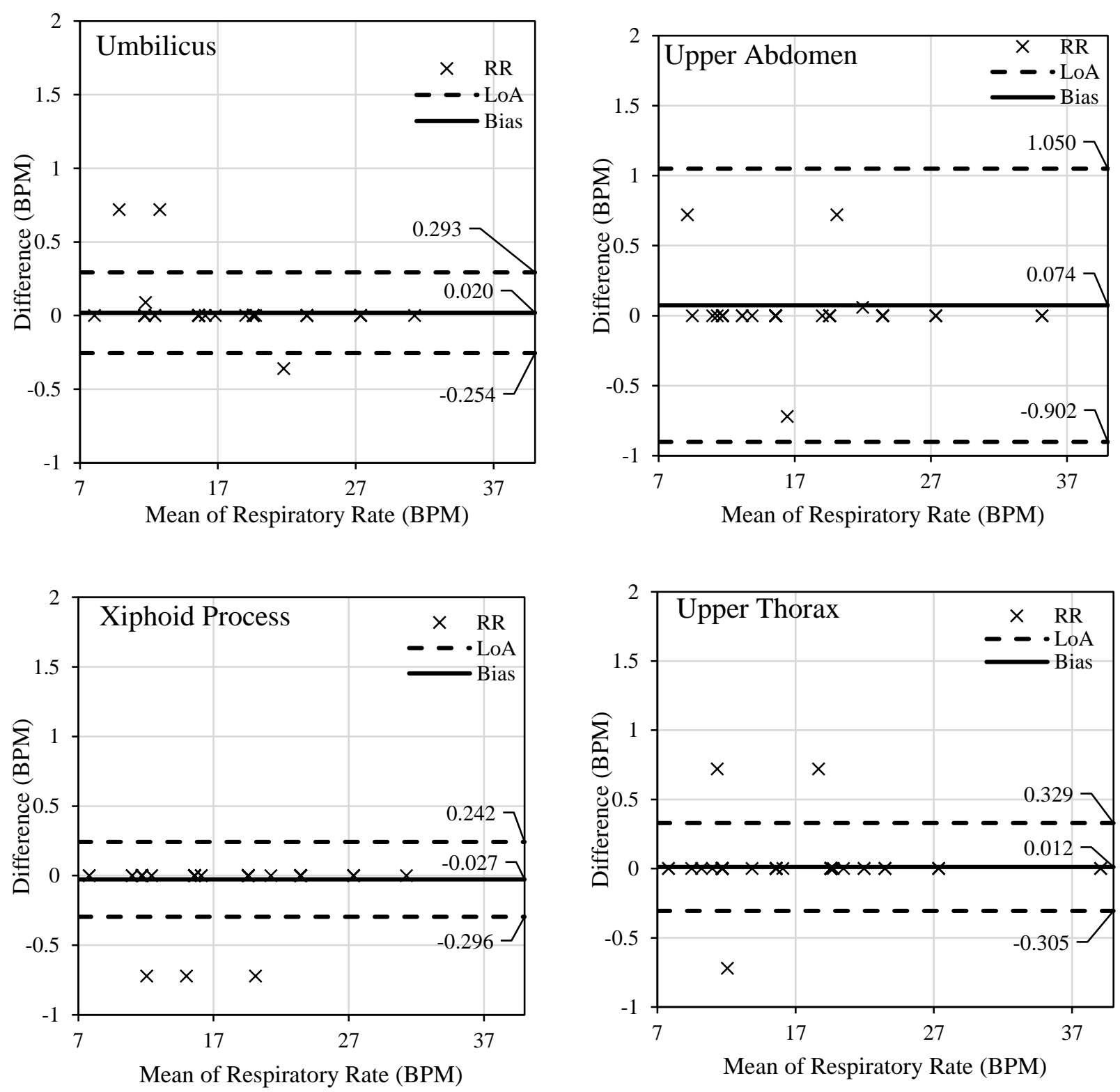


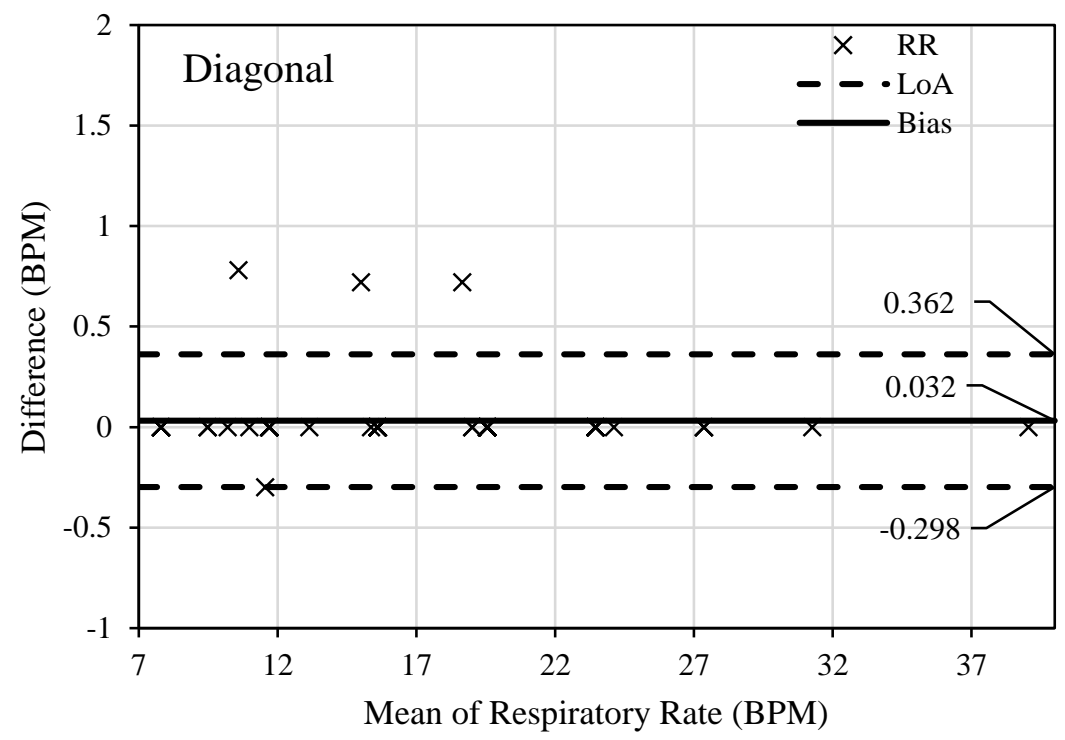

Figure 6. Bland-Altman analysis of the measured respiratory rate of the thirty adults at different measurements locations. Each data point represents the RR measured in each trial for the thirty subjects. The black continues line is the bias which is the average differences between the actual RR and the measured one while the dashed black lines are the limits of agreement (LoA) where $95 \%$ of the data lays in between.

Notice in Figure 5 that the RR measurements at xiphoid process and upper thorax had zero deviation from the reference sensor measurements on average where the measurements at the diagonal location had the largest deviation with 0.036 BPM on average. Finally, Bland-Altman analyses in Figure 6 show that the measurements at all the measurements locations lie within the agreement limits. The RR measurements from the IPSG and e-Health sensors had a very high correlation with regression coefficients between 0.9922 and 0.993 at the five measurement positions as shown in Table 1.

Table 1. The correlation between the RR measurements from the IPSG and e-Health sensors at different measurement locations.

\begin{tabular}{cccc}
\hline Location & Equation & R2 & p-value \\
\hline Umbilicus & $y=1.0076 x-0.1544$ & 0.9993 & $\mathrm{p}<0.001$ \\
Upper Abdomen & $y=0.9964 x-0.0096$ & 0.9992 & $\mathrm{p}<0.001$ \\
\hline
\end{tabular}




$\begin{array}{clcc}\text { Xiphoid Process } & y=0.9981 x+0.0679 & 0.9992 & \mathrm{p}<0.001 \\ \text { Upper Thorax } & y=0.9995 x-0.0027 & 0.9993 & \mathrm{p}<0.01 \\ \text { Diagonal } & y=1.0014 x-0.0562 & 0.9993 & \mathrm{p}<0.001\end{array}$

\section{Discussion}

Strain gauge sensors can be employed in the detection of the RR where it can be estimated by the detection of either the change in the volume of ribcage or the volume of the abdomen. The amount of volume change differs within the abdomen and thorax areas [18] which may affect the sensor's accuracy. Stretchable and wearable strain gauge sensors are more likely to be affected by the RR measurement's location due to their mechanical properties which highlights the need for further investigation on the performance of stretchable and wearable sensors for RR measurements at different locations.

\subsection{RR Measurements: Comparison with Other Sensors}

The RR measurements using the IPSG sensor were accurate compared with the sensors reported in the literature [8], [21], [38]-[43]. Moreover, the IPSG sensor was very accurate compared with the sensors reported in the literature [18], [21] with high regression coefficients as shown in Table 2.

Table 2. Comparison between the performance of different RR sensors reported in the literature and the IPSG sensor in this study.

\begin{tabular}{ccccc}
\hline Author & Sensor Type & Average Error & $\mathrm{R}^{2}$ & $\mathrm{p}$-value \\
\hline Hesse et al. [12] & force sensor & $-0.32 \pm 0.68 \mathrm{~Hz}$ & - & - \\
& & $0.14 \pm 0.01$ to & & \\
Chu et al. [21] & strain gauge sensor & $0.153 \pm 0.0115$ & $0.929-$ & $\mathrm{p}>0.05$ \\
& & BPM & 0.962 & \\
Kwak et al. [22] & strain gauge sensor & no apparent error & - & - \\
Chung et al. [38] & stretchable and wearable RR sensor & $0.3 \pm 0.95 \mathrm{BPM}$ & - & - \\
This study & IPSG sensor & $0 \pm 0.109$ to 0.082 & $0.992-$ & $\mathrm{p}>0.05$ \\
& & $\pm 0.109 \mathrm{BPM}$ & 0.993 & \\
\hline
\end{tabular}

\subsection{The Effect of The Measurement Location on RR}


Notice in Figure 6 that the measurements at the upper thorax location had the lowest means and SD of bias $(0.012 \pm 0.137 \mathrm{BPM})$ while the upper abdomen had the largest mean and SD of bias $(0.074 \pm 0.497$ BPM $)$. Furthermore, the measurements at the xiphoid process had the smallest limit of agreement range (0.538 BPM) while measurements at the upper abdomen had the biggest limit of agreement range (1.95 BPM). The RR measurements at the xiphoid process and the upper thorax were the most accurate ones compared with the reference sensor. Note that the dataset measurements at the upper abdomen area were the only one that had outliers could be related to an error during the measurement.

While the least accurate ones were taken at the upper abdomen followed by the diagonal location. Excluding the outlier from the regression analysis increases the regression coefficient to 0.9992 and make it more approximate to the other measurement's locations. It was reported in [18] that the abdomen area has the least volume change due to respiration while the thorax area had the biggest change (upper thorax followed by the xiphoid process) which justifies the accuracy of the measurements taken at the upper thorax and the xiphoid process. It can be then concluded that the amount of volume change had an insignificant effect on the RR detection by the IPSG sensor where the sensor was sensitive enough to detect the RR at any location in the abdomen and thorax areas. Finally, it can be concluded that the best location for the RR detection is either at the upper thorax (had the least bias and least difference) or at the xiphoid process (had the narrowest limits of agreements) which agree with [18] where they reported the largest volume change due to the respiration movement at these two locations.

It can be depicted from Figure 6 that the IPSG sensor was capable of detecting the RR at the five measurements locations accurately compared with the sensor reported in the literature [12], [19], [21], [22]. Moreover, the RR measurements by the IPSG sensors at the different locations in the abdomen and thorax areas had better regression coefficients compared with the sensors in the literature [18] at the same measurement locations as shown in Table 3. Finally, the test subject agreed that the RR measurements at the diagonal location were the most uncomfortable test while the measurements at the xiphoid process and the upper thorax were the most comfortable ones. 
Table 3. Comparison between the correlation coefficients of the RR measurements by the IPSG sensor and the strain gauge sensor in [18] at different measurements locations.

\begin{tabular}{lcc}
\hline Location & \multicolumn{2}{c}{ Correlation Coefficients } \\
\cline { 2 - 3 } & This Study & {$[18]$} \\
\hline Umbilicus & 0.9993 & 0.82 \\
Xiphoid & 0.9992 & 0.82 \\
Upper Thorax & 0.9993 & 0.85 \\
\hline
\end{tabular}

\subsection{Limitations and Future Work}

There were some limitations in this pilot study. Firstly, the IPSG was tested on thirty test subjects at different measurement body sites only while sitting and under normal breathing rhythms only. For further evaluation, the IPSG sensor should be tested while running, standing and sleeping under normal and abnormal breathing rhythms. Secondly, in this study the performance of the sensors was evaluated only at the middle of the abdomen and thorax areas as well as diagonal position where it is important to further evaluate the performance at the left and right positions of each location. The subjects were mainly healthy and young (age: $26.67 \pm 6.23$ years). Thirdly, in some pathological cases such as acute asthma and pneumonia, the RRs are hard to measure where the investigation of the RR sensor location during such cases is important [44], [45]. In future studies, various subjects (age, weight, thoracic circumference) with different physiological conditions such as walking and running- where high RR measurement is still difficult to achieve [6], [21]- could be considered. Moreover, the lack of full validation of eHealth airflow sensor is a limitation. However, in this pilot study, we only measured the normal RR range, therefore the effect of possible inaccuracy of e-Health sensor is quite limited. In the future, more reliable reference sensor should be used with more cases to cover a wide range of RR.

\section{Conclusions}

In this study, IPSG sensor showed good repeatability and accuracy in all the five locations for RR monitoring. The accuracy and the performance of the new sensor were comparable with commercial sensors, and better than the sensors reported in the literature. 
Acknowledgement: The authors would like to thank the Royal Academy of Engineering for Funding this project under grant Ref. IAPP1R2\100204. Moreover, the authors would like to thank the Industrial Research \& Development Fund in the Higher Council for Science \& Technology for their support for the project. In addition, the authors would like to thank Eng. Ali Al-Ghussein from the GJU Innovation and Entrepreneurship laboratory for providing the eHealth sensor.

\section{Conflicts of interest}

The authors confirm that there are no conflicts of interest to declare.

\section{References}

[1] D. Jarchi, D. Salvi, L. Tarassenko, and D. Clifton, "Validation of Instantaneous Respiratory Rate Using Reflectance PPG from Different Body Positions," Sensors, vol. 18, no. 11, p. 3705, Oct. 2018.

[2] C. Kelly, "Respiratory rate 1: why measurement and recording are crucial .," Nurs. Times, vol. 114, no. 4, pp. 23-24, 2018.

[3] J. F. Fie, M. Hendryx, and C. M. Helms, "Respiratory Rate Predicts Cardiopulmonary Arrest for Internal Medicine lnpatients," J. Gen. Intern. Med., vol. 8, no. 7, pp. 354-360, 1990.

[4] T. Flenady, T. Dwyer, and J. Applegarth, “Accurate respiratory rates count : So should you !," Australas. Emerg. Nurs. J., vol. 20, no. 1, pp. 45-47, 2017.

[5] P. B. Lovett, "The Vexatious Vital : Neither Clinical Measurements by Nurses Nor an Electronic Monitor Provides Accurate Measurements of Respiratory Rate in Triage," vol. 45, no. $1,2005$.

[6] C. Massaroni, A. Nicolò, D. Lo Presti, M. Sacchetti, S. Silvestri, and E. Schena, "Contactbased methods for measuring respiratory rate," Sensors (Switzerland), vol. 19, no. 4, pp. $1-47,2019$.

[7] D. Wu et al., "A wearable respiration monitoring system based on digital respiratory inductive plethysmography," 2009 Annu. Int. Conf. IEEE Eng. Med. Biol. Soc., pp. 4844- 
4847, 2009.

[8] P. J. Lee, "Clinical evaluation of a novel respiratory rate monitor," J. Clin. Monit. Comput., vol. 30, no. 2, pp. 175-183, Apr. 2016.

[9] S. Hamdani and A. Fernando, "The Application of a Piezo-Resistive Cardiorespiratory Sensor System in an Automobile Safety Belt," Sensors, vol. 15, no. 4, pp. 7742-7753, Mar. 2015.

[10] E. Koch and A. Dietzel, "Stretchable sensor array for respiratory monitoring," in 2017 19th International Conference on Solid-State Sensors, Actuators and Microsystems (TRANSDUCERS), 2017, pp. 2227-2230.

[11] J. Mead, "Measurement of the separate volume changes of rib cage and abdomen during breathing," vol. c, 2019.

[12] M. Hesse, P. Christ, T. Hormann, and U. Ruckert, "A respiration sensor for a chest-strap based wireless body sensor," in IEEE SENSORS 2014 Proceedings, 2014, vol. 2014Decem, no. December, pp. 490-493.

[13] Y. Liu, H. Wang, W. Zhao, M. Zhang, H. Qin, and Y. Xie, "Flexible, stretchable sensors for wearable health monitoring: Sensing mechanisms, materials, fabrication strategies and features," Sensors (Switzerland), vol. 18, no. 2, 2018.

[14] J. M. Abu-Khalaf, J. W. Park, D. J. Mascaro, and S. A. Mascaro, "Stretchable fingernail sensors for measurement of fingertip force," Proc. - 3rd Jt. EuroHaptics Conf. Symp. Haptic Interfaces Virtual Environ. Teleoperator Syst. World Haptics 2009, no. April 2009, pp. 625-626, 2009.

[15] J. M. Abu-khalaf, R. Saraireh, S. M. Eisa, and A. Al-halhouli, "Experimental Characterization of Inkjet-Printed Stretchable Circuits for Wearable Sensor Applications," Sensors, no. April, pp. 1-21, 2018.

[16] T. Q. Trung and N. Lee, "Flexible and Stretchable Physical Sensor Integrated Platforms for Wearable Human-Activity Monitoring and Personal Healthcare,” pp. 4338-4372, 2016. 
[17] S. G. Yoon, H. Koo, and S. T. Chang, "Highly Stretchable and Transparent Micro fl uidic Strain Sensors for Monitoring Human Body Motions,” 2015.

[18] H. Liu et al., "Reliability and validity of measuring respiration movement using a wearable strain sensor in healthy subjects," J. Phys. Ther. Sci., vol. 29, no. 9, pp. 1543$1547,2017$.

[19] N. T. Furtak and E. Skrzetuska, "Development of Screen-Printed Breathing Rate Sensors," FIBRES Text. East. Eur., vol. 6, no. 102, pp. 84-88, 2013.

[20] L. Leicht, P. Vetter, S. Leonhardt, and D. Teichmann, “The PhysioBelt : A Safety Belt Integrated Sensor System for Heart Activity and Respiration,” 2017 IEEE Int. Conf. Veh. Electron. Saf., pp. 191-195, 2017.

[21] M. Chu et al., "Respiration rate and volume measurements using wearable strain sensors," npj Digit. Med., vol. 2, no. 1, p. 8, 2019.

[22] Y. H. Kwak, J. Kim, and K. Kim, "Sleep monitoring sensor using flexible metal strain gauge," Jpn. J. Appl. Phys., vol. 57, no. 5S, p. 05GD03, May 2018.

[23] D. Kogan, A. Jain, S. Kimbro, G. Gutierrez, and V. Jain, "Respiratory Inductance Plethysmography Improved Diagnostic Sensitivity and Specificity of Obstructive Sleep Apnea," Respir. Care, vol. 61, no. 8, pp. 1033-1037, 2016.

[24] Y. Wu, D. Jiang, A. Bardill, S. De Gelidi, R. Bayford, and A. Demosthenous, "A high frame rate wearable EIT system using active electrode ASICs for lung respiration and heart rate monitoring," IEEE Trans. Circuits Syst. I Regul. Pap., vol. 65, no. 11, pp. 38103820, 2018.

[25] M. Adnane, Z. Jiang, S. Choi, and H. Jang, "Detecting specific health-related events using an integrated sensor system for vital sign monitoring," Sensors, vol. 9, no. 9, pp. 68976912, 2009.

[26] C. Voscopoulos, J. Brayanov, D. Ladd, M. Lalli, A. Panasyuk, and J. Freeman, "Evaluation of a Novel Noninvasive Respiration Monitor Providing Continuous Measurement of Minute Ventilation in Ambulatory Subjects in a Variety of Clinical Scenarios," Anesth. Analg., vol. 117, no. 1, pp. 91-100, Jul. 2013. 
[27] A. Al-Halhouli, L. Al-Ghussain, S. El Bouri, H. Liu, and D. Zheng, "Fabrication and Evaluation of a Novel Non-Invasive Stretchable and Wearable Respiratory Rate Sensor Based on Silver Nanoparticles Using Inkjet Printing Technology," Polymers (Basel)., vol. 11, no. 9, p. 1518, Sep. 2019.

[28] A. Al-Halhouli, H. Qitouqa, A. Alashqar, and J. Abu-Khalaf, "Inkjet printing for the fabrication of flexible/stretchable wearable electronic devices and sensors," Sens. Rev., vol. 38, no. 4, pp. 438-452, Sep. 2018.

[29] J. Abu-Khalaf, L. Al-Ghussain, and A. Al-Halhouli, "Fabrication of Stretchable Circuits on Polydimethylsiloxane (PDMS) Pre-Stretched Substrates by Inkjet Printing Silver Nanoparticles," Materials (Basel)., vol. 11, no. 12, p. 2377, 2018.

[30] J. M. Abu-khalaf et al., "Optimization of Geometry Parameters of Inkjet-Printed Silver Nanoparticle Traces on PDMS Substrates Using Response Surface Methodology,” Mater. (Basel, Switzerland), 2019.

[31] J. L. Bayo-Monton, A. Martinez-Millana, W. Han, C. Fernandez-Llatas, Y. Sun, and V. Traver, "Wearable Sensors Integrated with Internet of Things for Advancing eHealth Care," Sensors (Basel)., vol. 18, no. 6, pp. 1-18, 2018.

[32] R. Rákay, M. Višňovský, A. Galajdová, and D. Šimšík, “Testing Properties of E-health System Based on Arduino," J. Autom. Control. Vol. 3, 2015, Pages 122-126, vol. 3, no. 3, pp. 122-126, 2015.

[33] N. Petrellis, M. Birbas, and F. Gioulekas, "On the Design of Low-Cost IoT Sensor Node for e-Health Environments," 2019.

[34] A. Talpur, F. K. Shaikh, N. Baloch, E. Felemban, A. Khelil, and M. M. Alam, "Validation of Wired and Wireless Interconnected Body Sensor Networks," Sensors, vol. 19, no. 17, pp. 1-23, 2019.

[35] H. Liu, J. Allen, D. Zheng, and F. Chen, "Recent development of respiratory rate measurement technologies," Physiol. Meas., vol. 40, no. 7, p. 07TR01, Aug. 2019.

[36] A. Al-Halhouli, L. Al-Ghussain, S. El Bouri, F. Habash, H. Liu, and D. Zheng, "Clinical Evaluation of Stretchable and Wearable Inkjet-Printed Strain Gauge Sensor for 
Respiratory Rate Monitoring at Different Body Postures," Appl. Sci., vol. 10, no. 2, p. 480, Jan. 2020.

[37] K. Yeager, "LibGuides: SPSS Tutorials: Pearson Correlation."

[38] H. U. Chung et al., "Binodal, wireless epidermal electronic systems with in-sensor analytics for neonatal intensive care," Science, vol. 363, no. 6430, pp. 0-13, 2019.

[39] H. Turnbull et al., "Development of a novel device for objective respiratory rate measurement in low-resource settings," BMJ Innov., vol. 4, no. 4, pp. 185-191, Oct. 2018.

[40] J. Hernandez, Y. Li, J. M. Rehg, and R. W. Picard, "BioGlass: Physiological Parameter Estimation," Proc. 4th Int. Conf. Wirel. Mob. Commun. Healthc. - "Transforming Healthc. through Innov. Mob. Wirel. Technol., pp. 55-58, 2014.

[41] K. van Loon et al., "Wireless non-invasive continuous respiratory monitoring with FMCW radar: a clinical validation study," J. Clin. Monit. Comput., vol. 30, no. 6, pp. 797-805, Dec. 2016.

[42] Y. Guechi, A. Pichot, D. Frasca, F. Rayeh-Pelardy, J.-Y. Lardeur, and O. Mimoz, "Assessment of noninvasive acoustic respiration rate monitoring in patients admitted to an Emergency Department for drug or alcoholic poisoning," J. Clin. Monit. Comput., vol. 29, no. 6, pp. 721-726, Dec. 2015.

[43] C.-L. Shen et al., "Respiratory Rate Estimation by Using ECG, Impedance, and Motion Sensing in Smart Clothing," J. Med. Biol. Eng., vol. 37, no. 6, pp. 826-842, Dec. 2017.

[44] S. Kesten et al., "Respiratory Rate during Acute Asthma," Chest, vol. 97, no. 1, pp. 5862, Jan. 1990.

[45] R. Strauß, S. Ewig, K. Richter, T. König, G. Heller, and T. T. Bauer, "The prognostic significance of respiratory rate in patients with pneumonia: A retrospective analysis of data from 705928 hospitalized patients in Germany from 2010-2012," Dtsch. Arztebl. Int., vol. 111, no. 29-30, pp. 503-508, 2014.

\section{Appendix A}


Table 1. The average respiration rates of the thirty subjects at different positions.

\begin{tabular}{|c|c|c|c|c|c|c|c|c|c|c|}
\hline \multirow{2}{*}{ Subject } & \multicolumn{2}{|c|}{ Umbilicus } & \multicolumn{2}{|c|}{ Upper Abdomen } & \multicolumn{2}{|c|}{ Xiphoid Process } & \multicolumn{2}{|c|}{ Upper Thorax } & \multicolumn{2}{|c|}{ Diagonal } \\
\hline & IPSG & e-Health & IPSG & e-Health & IPSG & e-Health & IPSG & e-Health & IPSG & e-Health \\
\hline 1 & 11.7 & 11.7 & 15.6 & 15.6 & 11.7 & 11.7 & 11.7 & 11.7 & 7.8 & 7.8 \\
\hline 2 & 13.65 & 13.65 & 31.26 & 31.26 & 21.48 & 21.48 & 11.7 & 11.7 & 13.65 & 13.65 \\
\hline 3 & 29.31 & 29.31 & 27.36 & 27.36 & 27.36 & 27.36 & 27.36 & 27.36 & 29.31 & 29.31 \\
\hline 4 & 21.51 & 21.51 & 23.46 & 23.46 & 19.56 & 19.56 & 23.46 & 23.46 & 21.51 & 21.51 \\
\hline 5 & 19.53 & 19.53 & 13.65 & 13.65 & 13.65 & 13.65 & 11.7 & 11.7 & 13.65 & 13.5 \\
\hline 6 & 17.58 & 17.58 & 19.53 & 19.53 & 23.46 & 23.46 & 19.56 & 19.56 & 21.51 & 21.51 \\
\hline 7 & 15.63 & 15.63 & 11.7 & 11.7 & 11.7 & 11.7 & 11.7 & 11.7 & 9.75 & 9.75 \\
\hline 8 & 17.58 & 17.58 & 15.6 & 15.6 & 15.6 & 15.6 & 15.6 & 15.6 & 15.6 & 15.6 \\
\hline 9 & 19.56 & 19.56 & 15.63 & 15.63 & 11.7 & 11.7 & 15.6 & 15.6 & 13.65 & 13.65 \\
\hline 10 & 15.6 & 15.6 & 15.6 & 15.6 & 15.6 & 15.6 & 19.56 & 19.56 & 19.56 & 19.56 \\
\hline 11 & 15.63 & 15.63 & 15.6 & 15.6 & 15.6 & 15.6 & 13.65 & 13.65 & 15.6 & 15.6 \\
\hline 12 & 19.56 & 19.56 & 15.63 & 15.63 & 19.56 & 19.56 & 19.56 & 19.56 & 19.53 & 19.53 \\
\hline 13 & 17.58 & 17.58 & 15.63 & 15.63 & 11.7 & 11.7 & 11.7 & 11.7 & 11.7 & 11.7 \\
\hline 14 & 17.58 & 17.58 & 17.58 & 17.58 & 17.58 & 17.58 & 21.51 & 21.51 & 11.7 & 11.7 \\
\hline 15 & 17.58 & 17.58 & 13.65 & 13.65 & 11.7 & 11.7 & 13.65 & 13.65 & 15.63 & 15.63 \\
\hline 16 & 17.58 & 17.58 & 19.56 & 19.56 & 19.56 & 19.56 & 19.56 & 19.56 & 19.56 & 19.56 \\
\hline 17 & 17.58 & 17.58 & 17.58 & 17.58 & 19.56 & 19.56 & 17.58 & 17.58 & 21.51 & 21.51 \\
\hline 18 & 19.53 & 19.53 & 23.46 & 23.46 & 17.58 & 17.58 & 15.6 & 15.6 & 19.56 & 19.56 \\
\hline 19 & 17.58 & 17.58 & 15.6 & 15.6 & 9.75 & 9.75 & 9.75 & 9.75 & 9.75 & 9.75 \\
\hline 20 & 23.46 & 23.46 & 17.58 & 17.58 & 15.6 & 15.6 & 15.6 & 15.6 & 23.46 & 23.46 \\
\hline 21 & 17.58 & 17.58 & 19.56 & 19.56 & 21.51 & 21.51 & 21.51 & 21.51 & 23.46 & 23.46 \\
\hline 22 & 17.55 & 17.55 & 16.44 & 16.44 & 15.72 & 15.36 & 16.8 & 16.8 & 15 & 15.36 \\
\hline 23 & 20.85 & 20.67 & 20.85 & 21.24 & 21.93 & 21.57 & 21.21 & 21.21 & 21.21 & 21.57 \\
\hline 24 & 12.06 & 12.465 & 12.06 & 12.06 & 12.06 & 12.06 & 14.25 & 13.89 & 10.59 & 10.98 \\
\hline 25 & 10.59 & 10.95 & 9.12 & 9.48 & 10.98 & 10.98 & 10.23 & 10.23 & 9.84 & 9.84 \\
\hline 26 & 12.42 & 12.42 & 16.8 & 18.27 & 18.66 & 18.66 & 20.13 & 20.49 & 19.02 & 19.02 \\
\hline 27 & 12.42 & 12.42 & 12.225 & 12.225 & 12.42 & 12.06 & 10.59 & 10.95 & 11.31 & 11.31 \\
\hline 28 & 15.63 & 15.63 & 17.58 & 17.58 & 11.7 & 11.7 & 17.58 & 17.58 & 17.58 & 17.58 \\
\hline 29 & 23.46 & 23.46 & 19.53 & 19.53 & 23.46 & 23.46 & 21.51 & 21.51 & 23.46 & 23.46 \\
\hline 30 & 25.41 & 25.41 & 29.31 & 29.31 & 29.31 & 29.31 & 39.06 & 39.06 & 33.21 & 33.21 \\
\hline
\end{tabular}

